\title{
Téoros
}

Revue de recherche en tourisme

\section{Le cas des auberges}

\section{Normand Cazelais}

Volume 7, numéro 1, mars 1988

Cultures régionales et tourisme

URI : https://id.erudit.org/iderudit/1080432ar

DOI : https://doi.org/10.7202/1080432ar

Aller au sommaire du numéro

\section{Éditeur(s)}

Université du Québec à Montréal

\section{ISSN}

0712-8657 (imprimé)

1923-2705 (numérique)

Découvrir la revue

\section{Citer cet article}

Cazelais, N. (1988). Le cas des auberges. Téoros, 7(1), 32-34.

https://doi.org/10.7202/1080432ar d'utilisation que vous pouvez consulter en ligne.

https://apropos.erudit.org/fr/usagers/politique-dutilisation/ 


\section{Le}

\section{cas}

\section{des auberges}

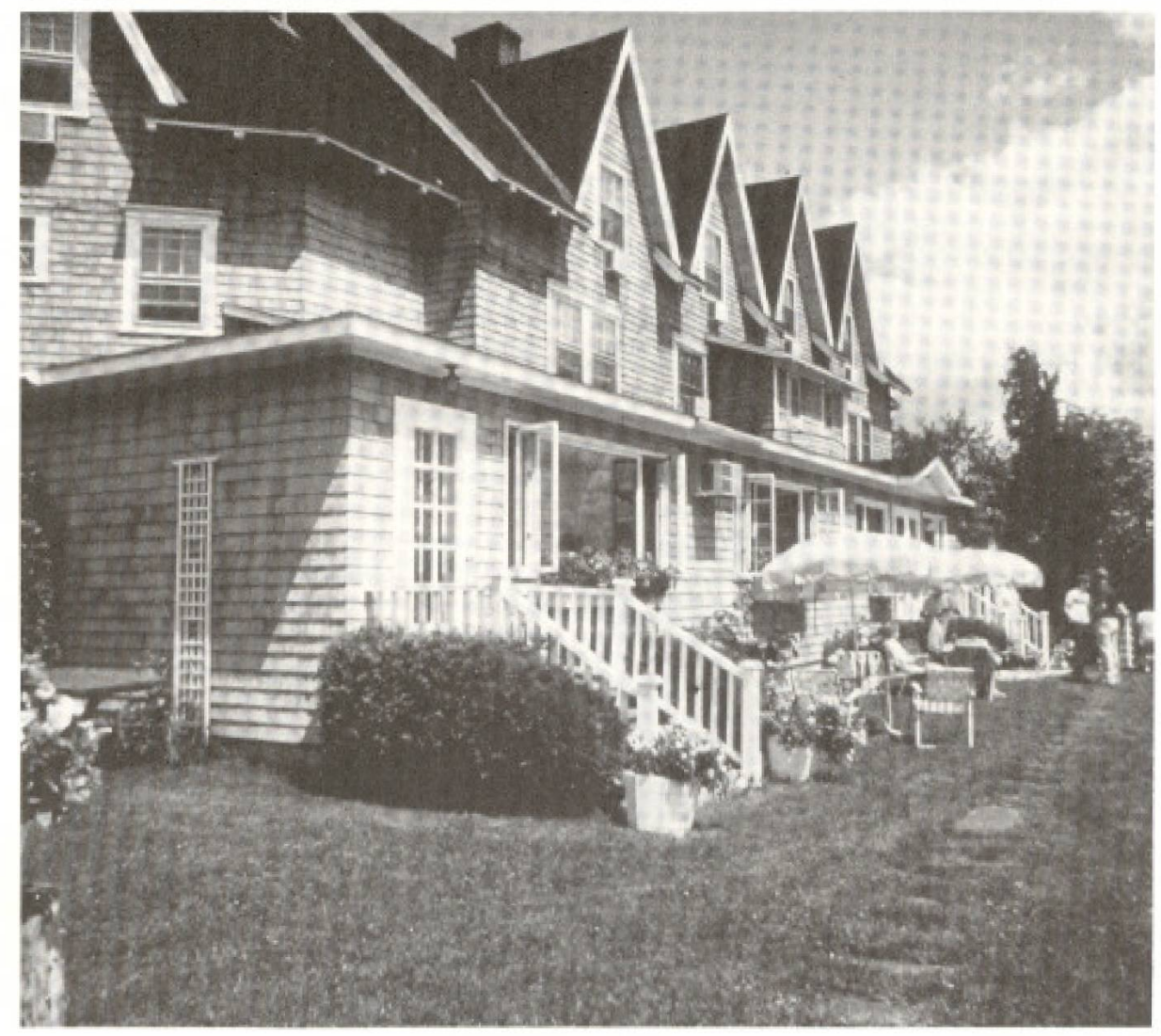

Cet exposé est un peu l'histoire d'un livre*** La préparation de ce livre, qui est un guide des auberges du Québec - donc une sélection personnalisée d'un certain nombre d'établissements -, a perm is dit ses auteurs de circonscrire cette relation si particulière qui existe entre les auberges et leurs régions respectives ainsi que l'évolution même du concept de l'auberge au Québec. Car on sait que, depuis une quinzaine d'années, les auberges ont connu un regain de popularité. Comme la société, elles ont évolué.

La première difficulté de ce livre a été de définir les auberges. Comme on peut le constater en se promenant un peu partoui au Québec, les Québécois n'en sont pas à leurs premiers abus de langage: il y a des hotels de 100 ou 200 chambres et des restaurants qui portent le nom d'auberges. Soulignons à ce propos que ni le ministère du Tourisme du Québec dans sa nouvelle Loi sur l'hôtellerie, ni l'Office de la langue française n'ont eru bon de définir le mot auberge.

*M. Normand Cazelais est géographe à l'Hydro-Ouébec, chroniqueur en tourisme et chargé de cours â I'UQAM.
Dans son introduction, notre livre disait que

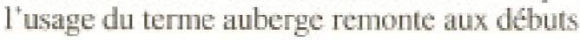

du XVIIe siècle et provient du provençal "aubergio". Son premier sens désigne une maison très simple, généralement à lă campagne où l'on trouve à manger en payant. Son second sens s'applique à un hôtel d'apparence champetre mais confortable et luxueux. Comme le sens, l'équipement et la fonction ont évolué, de même que l'opinion qu'on se fait d'une auberge. Les auberges furent pendant un certain temps associées à des équipements hôteliers de second ordre, ce qui explique, en partie, leur déclin - du moins au Québec - voici une trentaine d'années.

Or, pour nous, une auberge, c'était une certaine conception de l'hôtellerie.

Voici la définition que nous en avons donnée: "Nous entendons par auberges des établissements de quelques dizaines de chambres tout au plus, gérés sur les lieux par leurs propriétaires et caractérisés par un accueil chaleureux et personnalisé, par une cuisine de qualité et un décor agréable. Même si des chalets ou des unités de motel peuvent les compléter à l'occasion, les chambres d'une auberge sont - sauf en de très rares 
exceptions - dans le bâtiment principal et communiquent ainsi directement avec la salle à manger et la réception. Enfin, une auberge est un endroit où le tarif de l'hébergement inclut à la fois le gîte et le petit déjeuner (contrairement à un hôtel ou un motel) et également, si les clients le désirent, le repas du soir et parfois celui du midi (contrairement à un Bed and Breakfast).

Ainsi, il y a certains critères dits objectifs comme la taille de l'établissement, la présence du propriétaire sur les lieux, le service du petit déjeuner et d'au moins un autre repas dans une salle à manger, une architecture devant permettre une communication entre les chambres et le hall d'entrée, la salle à manger. La taille, entre autres, s'est avérée un critère important. Un exemple: La Sapinière, qui correspond beaucoup à l'esprit d'une auberge, n'a pas été retenue en raison de son grand nombre de chambres. D'autres établissements de qualité - pensons à l'Auberge de Bromont, au Manoir de BaieComeau - qui offrent un hébergement remarquable ne furent pas inscrits au guide parce qu'ils appartiennent à des entreprises ou à des holdings, parce que conséquemment, leurs propriétaires ne sont pas sur les lieux pour les gérer et accueillir les clients.

Il est en fait assez facile de référer à des tels critères pour établir une sélection. Mais interviennent aussi d'autres critères de nature plus subjective: ce sont, par exemple, la bonne bouffe (appuyée sur l'utilisation de produits frais et valorisant les recettes régionales), une architecture de qualité qui reflète les richesses du patrimoine. Ce ne sont pas là des critères qui s'insèrent aisément dans une grille d'évaluation comparative. Ce sont peut-être eux pourtant qui définissent vraiment ce qu'est une auberge. Ils illustrent également à quel point tout n'est pas si évident dans la définition et la sélection d'un produit touristique, surtout quand il s'agit d'auberges. Et ce sont justement ces critères plus ou moins impalpables qui expriment probablement avec plus d'acuité la relation très intime qui existe entre les auberges et un produit touristique régional ou encore avec l'identitế culturelle d'une région.

Développons le tout à l'aide de quelques exemples.

\section{L'exemple de la Gaspésie}

Le premier vient de la Matapédia. D'un établissement appelé La Coulée douce. Déjà, le nom est une sorte de poème. Les noms comptent pour beaucoup dans les auberges. La Coulé douce est à Causapscal, dans une ancienne résidence de prêtres qui sent encore l'encaustique. Située sur une butte, au milieu de la ville, près de la rivière qui murmure et gazouille.

C'est un oasis au milieu de la vallée, au milieu de la ville. Son propriétaire est né tout près, y est revenu après avoir travaillé dans les grands centres. Il fait aussi office de pourvoyeur de chasse et pèche. Il aime son coin de pays.
Un autre établissement a pour nom La Nor mandie. Cette auberge jouit sûrement du plus beau site au Québec: son jardin, son parterre, ses fenêtres donnent sur le Rocher Percé. $L a$ Normandie est un nom qui existe à Percé depuis toujours, ou presque. De génération en génération. Ce sont des jeunes qui ont pris la relève. L'établissement aujourd 'hui appartient à la catégorie haut-de-gamme. À Percé, qui est LE site touristique du Québec.

L Auberge du Parc, pour sa part, est située à Paspébiac, devant le banc de Paspébiac qui est un site historique. Elle loge dans l'ancienne résidence des Robin, cette famille qui a contrôlé durant des décennies l'économie de la Gaspésie. Elle est implantée dans un village dont les habitants s"appellent Delarosbil par exemple. Ce sont des descendants de Jerseyais et des Basques; ils forment une espèce d'illot culturel et social dans la péninsule gaspésienne.

Done, trois auberges en Gaspésie. La géographie de cette région, c'est un périmètre qui est aussi un littoral, c'est l'importance de la mer et de ses produits, c'est une population distribuée en chapelet où s'insèrent, comme je viens de le souligner, des illots culturels qu'on oublie parfois, surtout sur la côte de la baie des Chaleurs, avec des descendant: de Jerseyais, d'Irlandais, d'Ecossais, d'Acadiens. On dit la Gaspésie mais, en réalité, il y a plusieurs Gaspésie: celle du littoral. celle de la Matapédia (qu'on traverse trop rapidement en voulant se rendre à Percé). celle de la baie des Chaleurs, celle de l'extrémité de la pointe de Forillon, de Percé et de Gaspé. Sans compter toute la Gaspésie de la côte du Saint-Laurent. Ce sont autant de Gaspésie différente qu'on est porté à associer à un seul produit. Cette région, dotée d'une forte personnalité, mais marquée par un tourisme saisonnier, recèle l'un des hauts-lieux touristiques du Québec: Percé. C'est une région isolée, une région terminus: on ne passe pas à travers la Gaspésie pour aller ailleurs. C'est surtout une région en proie à des graves problèmes économiques, où conséquemment le tourisme est appelé à jouer un rôle-clé.

Les auberges de la région sont plus ou moins le reflet de ces caractéristiques générales. On y retrouve une variété des styles architecturaux présents dans les différentes parties de la Gaspésie. Au total, les auberges ne sont pas nombreuses en Gaspésie où foisonnent. au contraire, hôtels et motels. Elles sont comme la population, dispersées.

Ces auberges habitent des maisons pour la plupart inscrites dans l'histoire de la région. Revoyons l'Auberge du Parc, l'ex-résidence des Robin. À l'intérieur, le hall, l'escalier monumental, la salle à manger, le salon lui conferent l'apects d'un manoir: les riches de la région s'étaient donnés une résidence qui pouvait correspondre à leur rang. Au bord du Saint-Laurent, sur l'autre versant de la péninsule, le Château Lamoniagne, fut la maison du premier millionnaire de la Gaspésie. Et les actuels propriétaires se font un honneur de rappeler à leur clientèle que Monsieur Lamontagne fut, oui, le premier millionnaire de la Gaspésie.

L'auberge Au coin du banc appartient à Sidney Malloney. Il est depuis quinze ans êtabli à cet endroit. Mais, en fait, Sidney Malloney n'est pas encore revenu de l'île Bonaventure, de son île natale, où il a repris à la fin de la guerre un commerce qui appartenait à ses parents, oú il a donc été auber. giste. Selon une "bonne"t logique propre au Québec, on a voulu un jour faire un parc de cette île au large de Percé. Et, pour faire plus vrai, plus authentique, plus naturel sans doute, on a déménagé ses habitants sur le continent.

Sidney Malloney n'en est jamais revenu. II en parle encore. Dans son français cassé d'Irlandais qui a longtemps vécu sur l'île, il raconte sa jeunesse et les années de sa vie qui est encore là-bas. Il ne comprend pas pourquoi on l'a "chasséc" de son île, pourquoi on ne lui donne pas le droit, comme aux biologistes et aux fonctionnaires, de retourner y vivre; il ne comprend pas non plus pourquoi le gouvernement du Québec a récemment décidé de laisser sur place les décors du film Les fous de bassan recréés pour rappeler les habitants d'origine qu'on "a mis dehors",

Alors, Sidney Malloney regarde ses mains. Celles qui lui ont permis de construire, planche par planche, son auberge d'aujourd'hui dont le décor, un peu capharnaüm, est tout de même très joli, très agrếable. Et surtout remplie de souvenirs de sa vie antérieure. Car cet homme vit de façon déphasée. Et son auberge, c'est encore ce qui le rattache à une certaine réalité.

Le cas de I'Auberge du Parc est différent. Elle est effectivement à l'intérieur d'un parc, dont le terrain assez vaste descend en pente douce vers la mer. La propriétaire, madame Lemarquand, est une forte personnalité qui. voici quelques années, a décidé d'investir massivement dans son établissement. Elle y a, en particulier, développé un produit maintenant bien identifié à la Gaspésie, la thalassothérapie. Elle essaie en outre d'intéresser ses visiteurs au banc de Paspébiac, à l'histoire locale, à la réalité culturelle et sociale de ce village. De faire en sorte que les touristes s'arrêtent et découvrent une réalité différente de l'image gaspésienne stéréotypée.

Il m'est impossible de clore ce rapide survol des auberges de la Gaspésie sans parler du réseau La Barouche (dont font partie $L a$ Coulée douce, Le Coin du banc). Dans cette région éclatée, étirée, influencée par les defferlements du tourisme estival, ce réseau représente l'un des premiers efforts pour offrir un produit régional, axé sur une qualitế d'hébergement dans un cadre bien spécifique, axê sur une notion très élevée de l'accueil. Le réseau La Barouche, présent sur tout le pourtour de la Gaspésie, veut véhiculer un produit à la hauteur de l'image de la région. 
L'exemple des Cantons de l'Est

Les Cantons de l'Est forment une région de contact, à la fois physique et naturel, entre la plaine du Saint-Laurent et les montagnes, les Appalaches. Et une région de contact social et culturel: elle s'appelait The Eastern Townships à l'origine. Les Loyalistes et les francophones s'y sont succédés par vagues. Une dualité culturelle qui s'exprime dans ses paysages, dans son architecture, son occupation du sol, dans son cadastre. Elle est aussi une zone-frontière, une zone de contact avec le milieu américain. Alternant et mêlant espaces forestiers et montagneux, agricoles, urbains et touristiques, elle offre une variété d'attraits et de fonctions. Et, comme les Laurentides de Montréal, les Cantons de l'Est s'inscrivent à la périphérie et dans le prolongement du bassin de la métropole.

En fait, il s'agit ici de la partie occidentale des Cantons; a l'est et nord-est de Sherbrooke, il y a aussi Coaticook et Compton. Thetford-Mines et le Pays-de-l'Amiante qui présentent un tout autre visage, une tout autre réalité. Le tissu touristique de cette partie des Cantons de l'Est ne possède pas la continuité spatiale si caractéristique des Laurentides où l'on peut manger à Sainte-Adèle, faire des emplettes à Val-Morin, danser à SainteAgathe, skier à Saint-Sauveur, s'héberger à Sainte-Marguerite ou à Saint-Donat. Les Cantons de l'Est sont structures differemment: ses pôles, qu'il s'appellent Orford ou Sutton, sont plus etanches.

Les auberges de la région seront largement a l'image de cette organisation spatiale. Déjà, leurs noms, $L$ Aubergine, le Ripplecove, etc. traduisent un contexte particulier. Pour beaucoup, ces auberges vivent en d'anciennes maisons de ferme et répondent à la notion de rusticité encore attachée par certains au concept des auberges. Elles sont de plus tenues par des familles qui leur conferent une atmosphère "familiale".

Un autre genre et d'une catégorie supéricure,

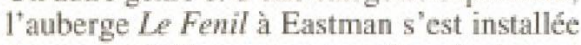
dans une vieille grange transformée du tout au tout. Quelques-unes, moins nombreuses. appartiennent à la catégorie des auberges haut-de-gamme et se sont implantées dans des anciens foyers de villégiature, comme l'Auberge de l'Étoile, à Magog, l'Auberge Hatley et le Manoir Hovey à North Hatley.

Ces derniers etablissements connaissent un problème que vivent certaines auberges de catégorie analogue en Montérégie: malgrế la distance, elles sont affectés par le "spread" urbain de Montréal qui s'étend dans. le sillage de l'autoroute. Elles sont plus ou moins situés en banlieue de leur clientèle, ce qui explique d'ailleurs qu'elles s'intéressent activement à la clientèle d'affaires et lui offre tous les services nécessaires.

Toutes ces auberges ont contribué à l'émergence d'un produit identifié à la rćgion. Quand on se promene par exemple à North Hatley, on voit ces résidences, longtemps de villègiature, qui furent bâties au tournant du siècle par des gens qui venaient d'aussi loin que la Virginie ou les Carolines, qui, contrairement ì nous, fuyaient les chaleurs estivales et venaient se réfugier en des lieux plus frais, considérés plus sains. Ils ont ainsi édifié un paysage, fait éclore une fonction de villégiature qui, sous une autre forme, après quelques décennies de déclin, a étét reprise par des auberges nommes Hatley, Manoir Hovey, en s"installant dans quelques-unes de ces chics demeures d'un autre âge.

D'autre part, dans les plis des Appalaches, les auberges Hatley, Hovey et Ripplecove, ancrées sur les rives du lac Massawippi, l'un des plus beaux de la région, se sont aussi associées dans l'implantation d'un produit touristique hivernal collectif: c'est le réseau de pistes de ski de randonnée Skiwippi. La formule fut reprise par la suite en d"autres régions, notamment dans les Laurentides et l'Outaouais. North Hatley n'est pas établie au pied de pistes de ski alpin. Pour promouvoir le ski de randonnce dans les pistes de leurs alentours, ces auberges ont conçu un forfait offrant à leur clientèle la possibilité de skier d'un établissement à l'autre et d'y loger à tour de rôle sans avoir à transporter leurs bagages, elles-mêmes s'cn chargeant.

\section{L'exemple de Charlevoix}

Charlevoix, pays de mer et montagne, possède des paysages exceptionnels. Longtemps en retrait, peu peuplé, c'est un pays compact, assez homogene, quoiqu'on oublie souvent qu'il y a plusieurs Charlevoix comme il y a plusieurs Gaspésie. En effet, il y a le Charlevoix du littoral, le Charlevoix du plateau. Et, sur le littoral, il y a le Charlevoix des anses et des baies, comme ill y a l'est de Charlevoix et l'ouest de Charlevoix. Les gens de Baie Saint-Paul ne sont pas ceux de La Malbaie et vice-versa; ainsi, l'an dernier, les premiers se sont sentis moins concernés par les problèmes survenus au Manoir Richelieu à Pointe-au-Pic/La Malbaie. Cette géographie, cette organisation sociale expliquent la région et sa rélité avec laquelle doit composer son développement touristique.

L'importance du fléve, des capitaines, des pilotes, des goélettes, de la navigation et du cabotage a activement contribué à façonner Charlevoix, à façonner son image. La région a aussi connu une tradition du grand tourisme international né au siècle dernier, comme l'a très bien rappelé l'exposition de Philippe Dubé sur la villégiature en Charlevoix. Un grand tourisme qui s'est notamment développé autour des stations chics de La Malbaie (qu'on appelait Murray Bay), de Pointe-au-Pic, de Cap-ă-l'Aigle, autour de personnages importants comme le président américain Taft, venu inaugurer en personne le golf de Pointe-au-Pic. Charlevoix a été une destination, un lieu de villégiature et de tourisme très important, très recherché.

Aujourd'hui, Charlevoix connaît une concentration d'auberges remarquable. Remarquable par sa diversité, par son nombre et par son rayonnement. Le mouvement de renais- sance des auberges au Québec s'est d'ailleurs amorcé en Charlevoix et même autour d'une personne qui peut en etre, pour une large part, considéré comme l'instigateur. Il s'agit de Ferdinand Tremblay, propriétaire de $\mathrm{I}^{+}$Auberge des Peupliers à Cap-à-l'Aigle, qui s'est avéré le pionnier, celui qui a donné un second souffle aux auberges non seulement de Charlevoix mais de tout le Québec en leur proposant, par l'exemple de son établissement et de son action, un idéal d'accueil, une qualité supérieure de décoration, de confort et de cuisine, un attachement également aux racines culturelles régionales.

L'exemple de Ferdinand Tremblay a été suivi. Dans Charlevoix et dans tout le Québec. Ainsi, Charlevoix à lui seul compte aujourd'hui plus de 30 auberges dont près de la moitié sont des auberges haut-de-gamme pouvant concurrencer, en termes de service, d'accueil, de cuisine et de confort, les grands hôtels qui ont cependant des tarifs plus élevés. Il faut aussi rappeler que Charlevoix était devenue, après le déclin des croisières et de la grande villégiature, après l'essor de l'automobile et du réseau routier, une région qu'on ne faisait que traverser. Ce n'était plus une destination. On s'y arrêtait le temps de quelques soupirs, le temps d'admirer - vite fait - le paysage, avant de continuer vers le Saguenay, la Cóte-Nord, le Bas SaintLaurent ou Quebec. Voici quinze ans, on n'arrêtait plus dans Charlevoix: ses visiteurs y séjournaient en moyenne moins d'une journée. Beaucoup de touristes passaient dans Charlevoix mais Charlevoix n'en profitait pas. La région en éprouvait même certains inconvénients (ex.: attentes aux traversiers, tronçons de route engorgés quelques weekends par année).

Les auberges ont activement contribué à refaire de Charlevoix une destination, une région touristique de séjour et recherchée comme telle. Le cas de Charlevoix illustre éloquemment l'identification pouvant s'établir entre une région et ses auberges. Un dernier exemple: lors de l'attribution des Grands Prix Québécois du Tourisme en 1986, les auberges de Charlevoix figuraient parmi les deux finalistes nationaux pour le développement. Dans toutes les autres catégories, les finalistes et aussi les candidats étaient des personnes ou des entreprises individuelles (hôtels, centres de ski, etc.). Les auberges de Charlevoix constituaient alors le seul candidat à s'être présenté et à être sélectionné sous un label à la fois collectif et régional. Toute l'argumentation de leur dossier s'appuyait sur cette cohésion interne et sur leur relation intime avec le vie touristique mais aussi avec la vie culturelle de leur région.

En ce Québec qui a encore largement besoin d'action concertée et d'enracinement, c'était un fait hautement original, c'était une première. Qui aura certainement des suites. $f$

\footnotetext{
"CAZELAIS, Normand et COULON, Jacques, Sejours dans les auberges du Québec, Éditions de I'Homme, 1965 .
} 